\title{
Characterization of articular chondrocytes isolated from 211 osteoarthritic patients
}

\author{
Matteo Laganà - Chiara Arrigoni - Silvia Lopa • \\ Valerio Sansone $\cdot$ Luigi Zagra $\cdot$ Matteo Moretti • \\ Manuela Teresa Raimondi
}

Received: 11 December 2012/ Accepted: 22 March 2013

(C) Springer Science+Business Media Dordrecht 2013

\begin{abstract}
We analyzed specific features of chondrocytes as cellular yield, cell doubling rates and the dependence between these parameters and patientrelated data in a set of 211 osteoarthritic (OA) patients undergoing total joint replacement. For each patient the data available were joint type, age and gender. Knee samples chosen randomly among all biopsies were graded according to ICRS score. Patients' age ranged between 30 and 90 years with a mean age of $66 \pm 9.7$ years. Patients were divided into age classes and statistically significant differences in proliferation rate at passage 1 were found between chondrocytes derived from young and old donors, with the last ones characterized by a lower proliferation rate. A similar
\end{abstract}

Chiara Arrigoni andSilvia Lopa contributed equally to this work.

Matteo Moretti and Manuela Teresa Raimondi contributed equally to this work.

M. Laganà · M. T. Raimondi $(\square)$

LaBS, Department of Structural Engineering,

Politecnico di Milano, Piazza Leonardo da Vinci 32,

20133 Milan, Italy

e-mail: manuela.raimondi@biomed.polimi.it

C. Arrigoni $\cdot$ S. Lopa

Cell and Tissue Engineering Laboratory, Gruppo

Ospedaliero San Donato Foundation, Milan, Italy

V. Sansone

Orthopaedic Department, IRCCS Galeazzi Orthopaedic

Institute, University of Milan, Milan, Italy trend was observed for proliferation rate at passage 2 . For all the samples, cellular yields ranged between 0.1 and 5.5 million cells/g of tissue. No significant correlation was observed between the level of cartilage degeneration (ICRS score) and cellular yield and proliferation rates. However, in samples with a high degree of cartilage degeneration (ICRS score 4) the cellular yield was lower compared to the other three groups (ICRS scores 1-3). In this study we performed a systematic characterization of basic parameters of chondrocytes originating from a wide group of OA patients. Considering the use of autologous chondrocytes in chondral treatments, the characterization of cell basic features may represent an important step to determine the quality of the cell source which is a major determinant in the outcome of cell-based therapies.

Keywords Osteoarthritis - Articular chondrocyte · Age $\cdot$ Yield $\cdot$ ICRS score

L. Zagra

Hip Department, IRCCS Galeazzi Orthopaedic Institute, Milan, Italy

M. Moretti

Cell and Tissue Engineering Laboratory, IRCCS Galeazzi Orthopaedic Institute, Milan, Italy 


\section{Introduction}

Osteoarthritis (OA) is a highly disabling pathology which is worldwide investigated by the scientific community due to its increasing diffusion. This agedependent degenerative disease induces the progressive damage of articular cartilage and subchondral bone and can eventually lead to the complete loss of joint functionality (Aigner et al. 2004; van der Kraan and van den Berg 2008). The combination of pharmacological and non-pharmacological therapies has been proved to be useful for the treatment of early- and middle-stage OA patients, but a successful clinical outcome is not granted when treating late-stage OA patients (Zhang et al. 2008). In this category of patients, the severe pain and the functional limitations caused by advanced OA degeneration are currently resolved mainly by joint replacement surgery.

In the last two decades, "advanced therapies" have been intensely investigated in developed countries, with the aim to find a biological solution to several degenerative pathologies. In cartilage tissue engineering, a possible strategy for an autologous approach would be to isolate chondrocytes from a cartilage biopsy, to expand the cells and possibly cryopreserve them for future re-intervention. Expanded chondrocytes are then seeded on a biomaterial and the cellularized construct is finally implanted back to the patient (Roseti et al. 2011). Cartilage tissue engineering applied to OA patients appears to be in contrast with the controversial opinion that healthy autologous chondrocytes or mesenchymal stem cells are necessary to obtain healthy cartilage in vitro (Tran-Khanh et al. 2005; van der Kraan and van den Berg 2008; Wang et al. 2006). Recent works, however, have demonstrated the possibility to use human OA chondrocytes to obtain autologous engineered cartilage with properties similar to those of the constructs obtained from non-OA chondrocytes (Carossino et al. 2007; Cavallo et al. 2010; Dehne et al. 2009; Tallheden et al. 2005). Moreover, a recent work (Neri et al. 2011) on human OA articular chondrocytes characterized their genetic stability during long term expansion, and demonstrated the safety of this cell type for autologous advanced therapies. Since the quality of cells used to generate the engineered tissue greatly influences the outcome, a characterization of OA chondrocytes in a wide patient group can help in extending the validity of the reported results.
In this work we analyzed specific features of chondrocytes collected from 211 osteoarthritic patients undergoing total joint replacement. The characteristics investigated were cellular yield and cell doubling rates, and the dependence between these parameters and some patients-related data.

\section{Materials and methods}

Chondrocyte isolation, expansion and data collection

Samples of adult human articular cartilage were harvested from subjects undergoing hip and knee routine arthroplasty procedures. With the patient's informed consent and in respect to the privacy law, the patient age, gender and joint type were recorded. Based on evaluation by orthopaedic surgeons, random knee samples were also graded from scores 1 to 4 using the ICRS protocol (Kleemann et al. 2005), where higher scores indicate higher levels of cartilage degeneration. Minced cartilage fragments $\left(2-3 \mathrm{~mm}^{2}\right)$ were weighted and chondrocyte isolation was carried out by cartilage digestion in $0.15 \%$ collagenase type II (Worthington) performed for $22 \mathrm{~h}$ at $37^{\circ} \mathrm{C}$, as previously described (Jakob et al. 2003).

Viable cells were counted by trypan blue dye exclusion. Freshly isolated chondrocytes (P0) were plated for expansion at a density of $1 \times 10^{4}$ cells $/ \mathrm{cm}^{2}$ for the first passage (P1) and cultured with complete proliferation medium (CM) containing Dulbecco's Modified Eagle Medium (DMEM) supplemented with $10 \%$ fetal bovine serum (FBS, Lonza), $1 \mathrm{mM}$ sodium pyruvate, $100 \mathrm{mM}$ HEPES buffer, $100 \mathrm{U} / \mathrm{ml}$ penicillin, $100 \mu \mathrm{g} / \mathrm{ml}$ streptomycin, $0.29 \mathrm{mg} / \mathrm{ml}$ L-glutamine, 1 ng/ml TGFb1, 5 ng/ml FGF-2 (PeproTech) (Barbero et al. 2003). Where not otherwise specified, Invitrogen products were used. For the second passage (P2) cells were plated at a density of $0.5 \times 10^{4}$ cells $/ \mathrm{cm}^{2}$. Cell doubling rate $(d)$ was calculated as

$\mathrm{d}=\frac{\log _{2}\left(\frac{N}{N_{0}}\right)}{\mathrm{t}}$

where $N$ and $N_{0}$ are the final and initial cell number, respectively, and $t$ is the expansion time. The cellular yield was calculated as the ratio between the viable cell number at $\mathrm{P} 0$, expressed in millions of cells, and 


\begin{tabular}{ccc}
\hline & $\begin{array}{c}\text { Males } \\
{[\%]}\end{array}$ & $\begin{array}{c}\text { Females } \\
{[\%]}\end{array}$ \\
\hline Hip patients & $42 \%$ & $58 \%$ \\
\hline Knee patients & $12 \%$ & $88 \%$ \\
\hline Total patients & $31 \%$ & $69 \%$ \\
\hline
\end{tabular}

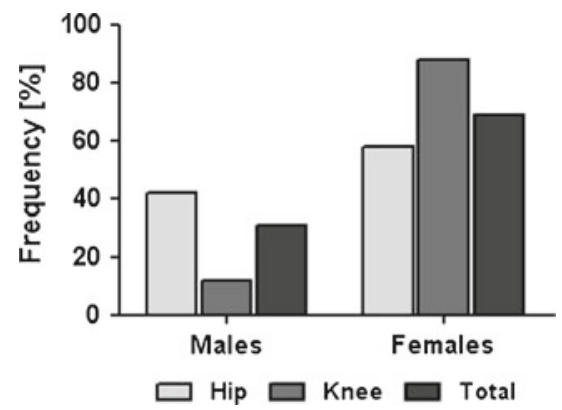

Fig. 1 Frequency distribution of patient gender. The patients, all affected by osteoarthritis, were subjected to total hip or knee replacement

the wet weight of the cartilage fragments, expressed in grams.

Data processing and statistical analysis

The software Graph Pad Prism ${ }^{\circledR}$ was used to order and process the data. For each patient, the available data were joint type, patient age, patient gender, ICRS grading, cellular yield, doubling rate at $\mathrm{P} 1$ and at $\mathrm{P} 2$. Mean and standard deviation were calculated for each parameter and relative normalized frequency (2) histograms were obtained. The normalized frequency was calculated as

$n f=\frac{f}{S_{c} \times N_{s}}$

where $f$ is the frequency of the data inside the considered class, $S_{c}$ is the spacing of the class and $N_{s}$ is the sample size (132 patients for the hip group, 88 patients for the knee group). Relative normalized frequency histograms were also obtained for the scored knee cartilage samples. For all the relative normalized frequency histograms the Poisson distribution was calculated.

Non normal distribution of data was determined with a D'Agostino \& Pearson normality test. Correlation between patients' age, cellular yield, and proliferation rate at $\mathrm{P} 1$ and at $\mathrm{P} 2$ was determined calculating the Spearman coefficient $\mathrm{r}$ and considered significant for $p<0.05$. To compare independent groups, Kruskal-Wallis test followed by Dunn's multiple comparison test was performed and differences were considered significant for $p<0.05$.

To better evaluate age-related differences in cellular yield and proliferation rate, patients were grouped in the following age classes: $\leq 60,65-70, \geq 75$ years, composed of the same number of patients $(n=47)$. Four years gaps between classes were left to avoid overlapping.

Box-plots were used for the visualization of the main parameters of the data statistical distribution both for age classes and for ICRS score groups (minimum, 25th percentile, 75 th percentile, maximum).

\section{Results}

Patients' gender distribution showed that $42 \%$ of hip arthroplasties were performed on males and $58 \%$ on females (Fig. 1). For knee arthroplasties the percentage of male versus female patients was $12 \%$ versus $88 \%$. Considering both arthroplasty types together, female patients were $69 \%$ of the total. Patients' age ranged between 30 and 90 years and the frequency histograms showed a mean age of 66 years, both for knee and hip patients (Fig. 2a-c). The ICRS grading system was applied to samples of knee cartilage randomly chosen among the donors, to classify the samples from scores 1 (low damage) to 4 (high damage). The mean age of patients did not differ significantly between scores and ranged from 65 to 69 years, as shown in frequency distribution graphs in Fig. 2d-g.

Cellular yield values ranged between 0.1 and 5.5 million cells/g of tissue, with a mean of $1.85 \pm$ 1.37 and $1.62 \pm 1.08$ million cells/g of tissue for hip and knee samples, respectively (Fig. 3a-c). For the different ICRS scores, mean values of cellular yield were similar up to score 3 (about 1.4 million cells/g of tissue), whereas it was lower for highly degenerated tissue (score 4, 0.9 million cells/g of tissue).

The analysis of the correlation between patients' age, cellular yield and proliferation rate at P1 and at P2 

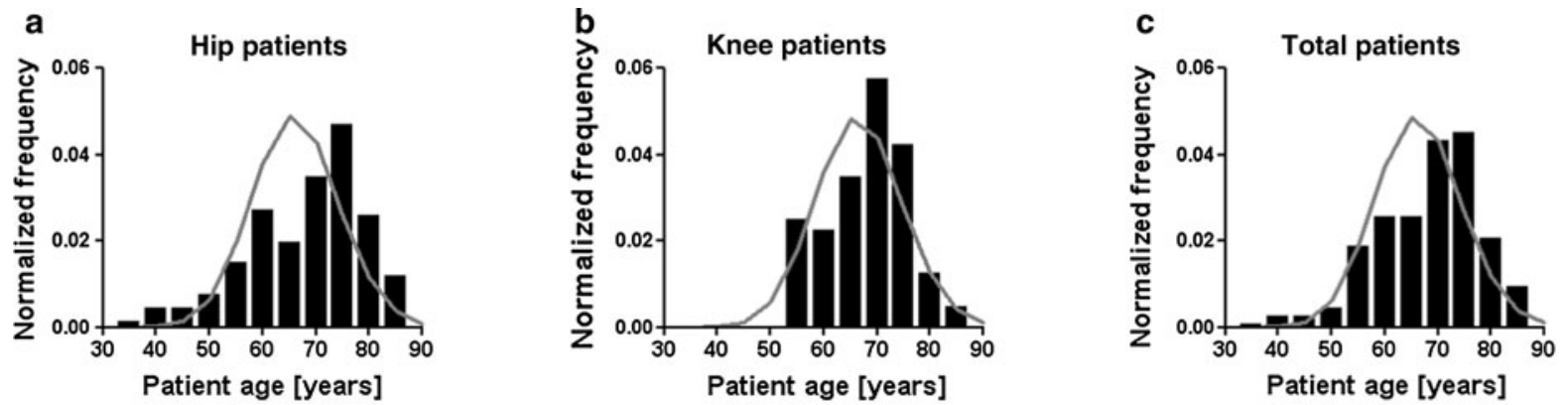

d

Knee patients
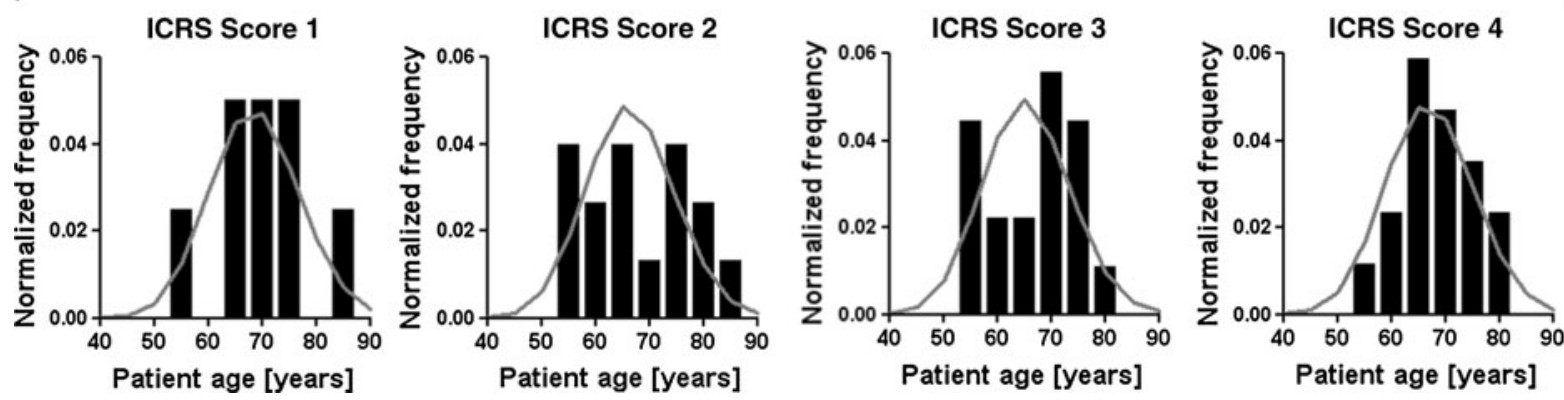

Fig. 2 Relative normalized frequency distribution obtained for increasing classes of patient age for the hip (a), knee (b), total (c) and increasing ICRS scores $(\mathbf{d}-\mathbf{g})$. The frequency histograms are approximated by Poisson's distribution

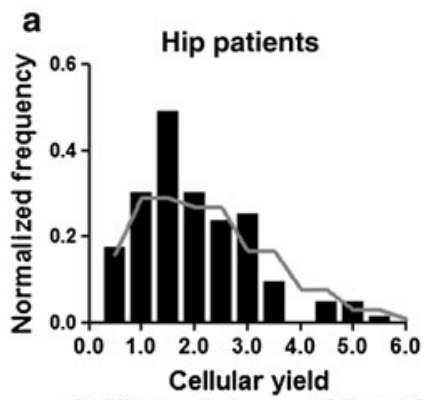

[million cells/gram of tissue]

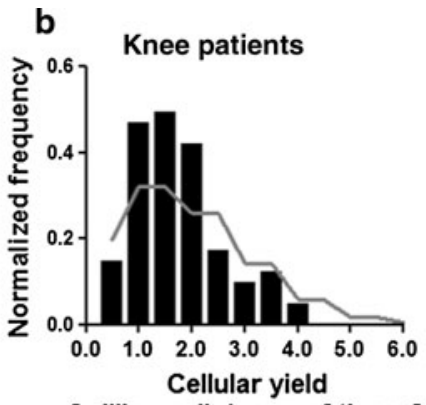

[million cells/gram of tissue]

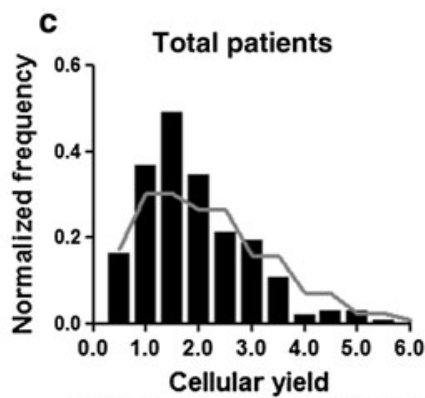

[million cells/gram of tissue]

d

Knee patients
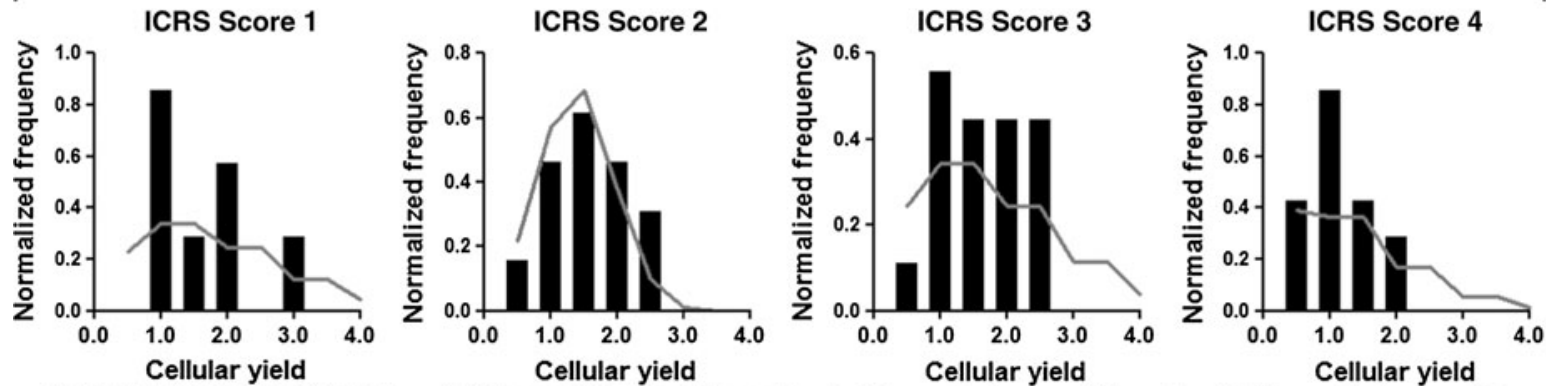

[million cells/gram of tissue] [million cells/gram of tissue] [million cells/gram of tissue]

[million cells/gram of tissue]

Fig. 3 Relative normalized frequency distribution obtained for increasing classes of cellular yield for the hip (a), knee (b), total (c) and increasing ICRS scores $(\mathbf{d}-\mathbf{g})$. The frequency histograms are approximated by Poisson's distribution 

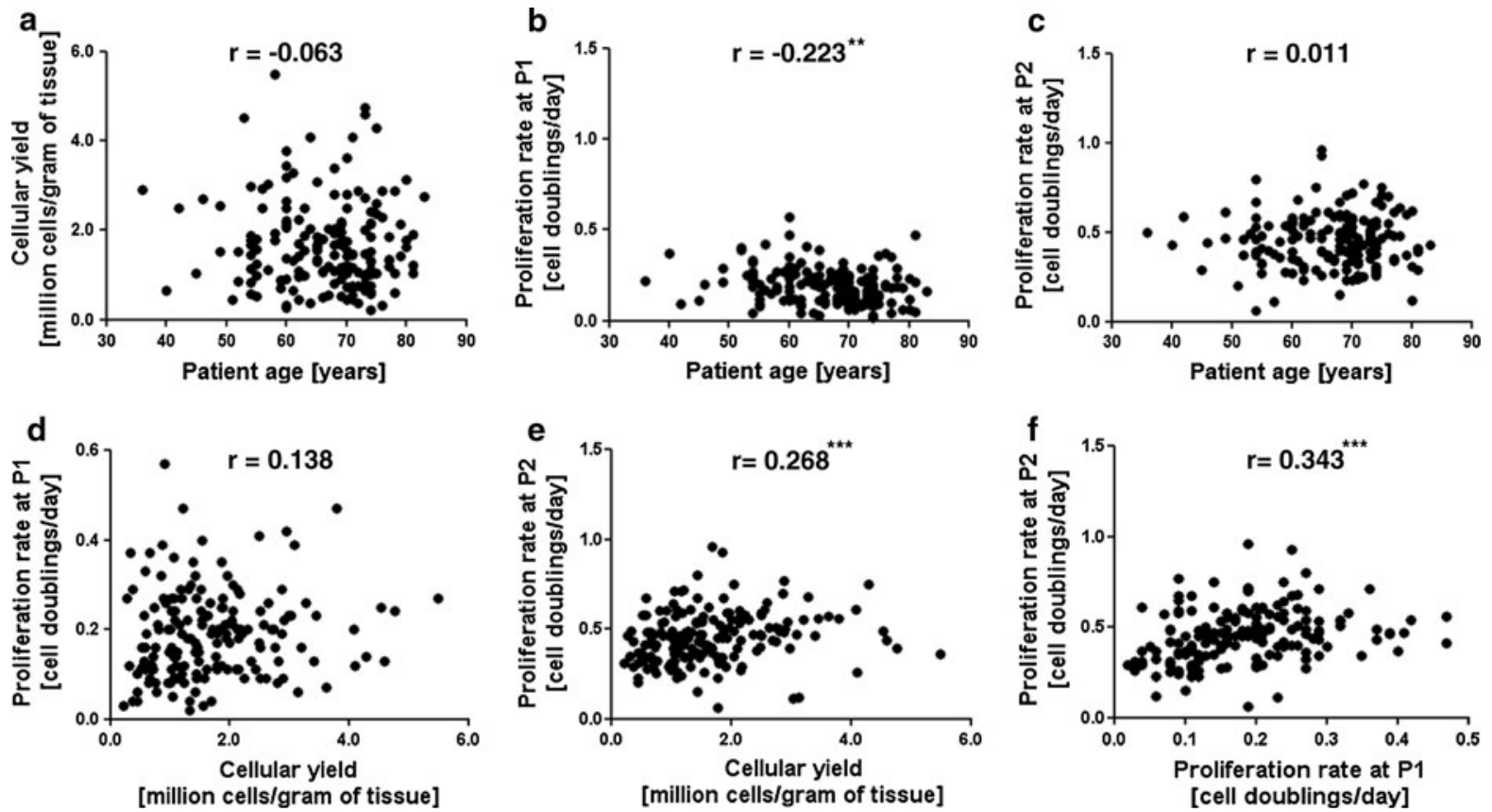

Fig. 4 Correlations among individual data groups. a Cellular yield versus patient age, $\mathbf{b}$ proliferation rate at $\mathrm{P} 1$ versus patient age, $\mathbf{c}$ proliferation rate at $\mathrm{P} 2$ versus patient age, $\mathbf{d}$ proliferation rate at $\mathrm{P} 1$ versus cellular yield, $\mathbf{e}$ proliferation rate at $\mathrm{P} 2$ versus

cellular yield and $\mathbf{f}$ proliferation rate at $\mathrm{P} 2$ versus proliferation rate at $\mathrm{P} 1$. Correlation coefficients (Spearman $\mathrm{r}$ ) are indicated in each graph. $* * p<0.01, * * * p<0.001$

are reported in Fig. 4 with the corresponding correlation coefficients (Spearman r). Significant correlations were observed between age and doubling rate at P1 $(\mathrm{r}=-0.223, p<0.01)$, showing a decrease of doubling rate at $\mathrm{P} 1$ with increasing age, and between doubling rate at $\mathrm{P} 2$ and at $\mathrm{P} 1(\mathrm{r}=0.343, p<0.001)$, demonstrating a trend between cell growth at both passages. A significant correlation was found also between cellular yield and proliferation rate at P2

( $\mathrm{r}=0.268, p<0.001)$, suggesting that cells deriving from samples with a higher cellular yield, retain a greater proliferation potential during expansion.

Pair wise comparison of data, grouped on the basis of patient age classes, homogeneous in size, are shown in Fig. 5. The average cellular yield did not significantly differ between age classes (Fig. 5a). We found statistically significant differences on proliferation rates at P1 between the age class $\leq 60$ as compared to
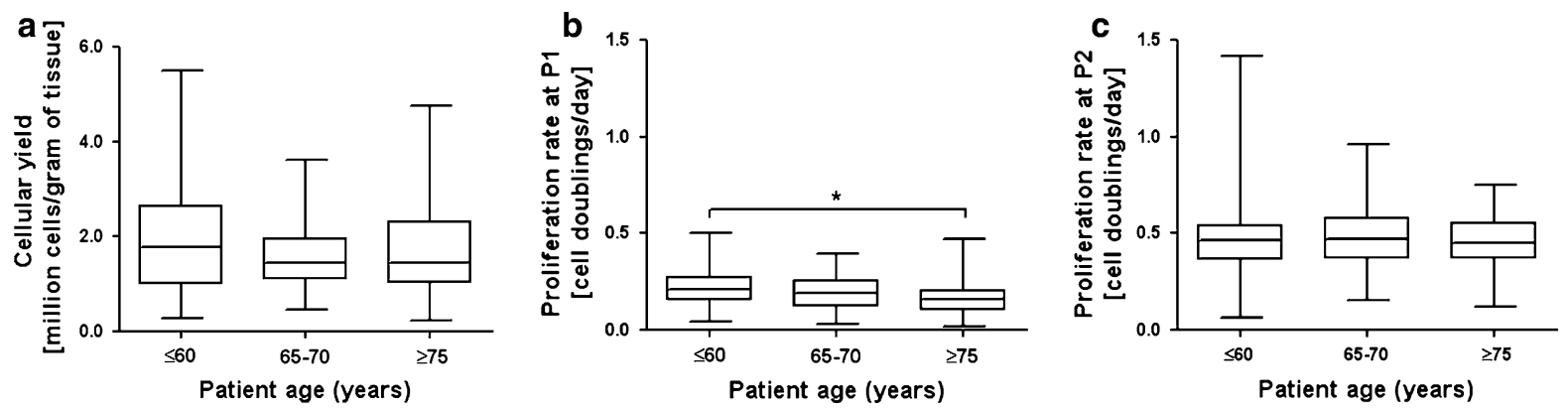

Fig. 5 Box plots showing pair-wise comparisons of grouped data pertaining to patient age and cellular yield classes homogeneous in size. a Cellular yield versus patient age, b proliferation rate at $\mathrm{P} 1$ versus patient age, $\mathbf{c}$ proliferation rate at $\mathrm{P} 2$ versus patient age. The values delimiting the box in the box plots are the 25th and 75th percentiles of the data distribution. $* p<0.05$ 

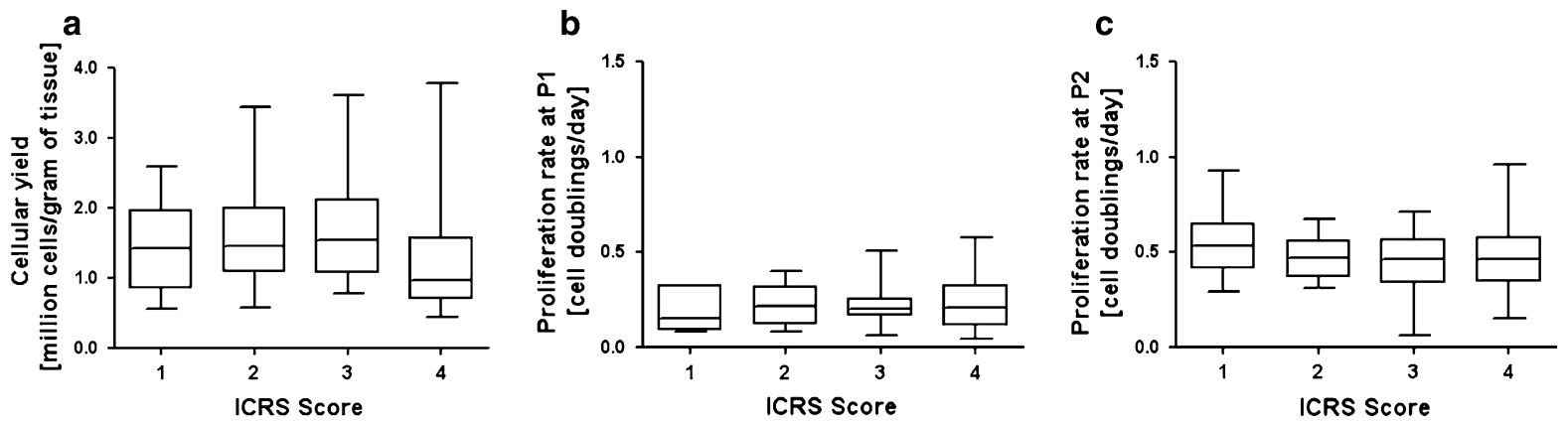

Fig. 6 Box plots $(\mathbf{a}-\mathbf{c})$ for the ICRS-scored knee cartilage samples. The values delimiting the box in the box plots are the 25 th and 75 th percentiles of the data distribution

the $\geq 75$, which was characterized by the lowest proliferation rate (Fig. 5b). Proliferation rates at $\mathrm{P} 2$ were higher than at $\mathrm{P} 1$, independently from the patient age (Fig. 5c).

No significant correlation was found among ICRS scores, cellular yields and proliferation rates at $\mathrm{P} 1$ and P2, as shown in Fig. 6. However, as the cartilage damage level increased, the mean cellular yield slightly decreased, whereas the proliferation rates did not differ with increasing scores. As already observed for data on hip and knee population, proliferation rates at $\mathrm{P} 2$ were higher than the rates at $\mathrm{P} 1$.

\section{Discussion}

OA is fully recognized as an age-dependent degenerative disease (Loeser et al. 2012). Considering the high and increasing life expectancy in developed countries, osteoarthritis is assuming an important social effect. Coherently, countermeasures to OA such as cellular therapies are intensely investigated (Goldring and Goldring 2007). In this study, adult human articular chondrocytes were isolated from anatomical specimens discarded during total joint replacement, with the aim to characterize them in view of their potential use as source for autologous cell therapy. More than 200 OA patients were included in this analysis, which required, for our institution, a period of 4 years for data collection. Accordingly with literature (Cicuttini et al. 2003; Musumeci et al. 2011; Wluka et al. 2001) we found that the majority of patients subjected to total joint replacement were postmenopausal women. The age of patients in our analysis ranged from 30 to 90 years old, but the frequency distributions showed that the highest amount of patients belonged to the range 65-70 years. The pair-wise analysis of data on cellular yield and proliferation rates showed that the patient age is not statistically correlated to the cellular yield, as in (Bobacz et al. 2004), but was negatively correlated with the proliferation rate at $\mathrm{P} 1$, which followed a trend analogue to the proliferation rate at P2. As already reported in the literature (Barbero et al. 2004) for healthy chondrocytes, the most relevant reduction in cellular yield is observed between patients younger than 40 years and older patients, then with increasing age this feature remains almost constant. $75 \%$ of our patients are older than 63 years: this could explain the lack of significant influence of patient age on cellular yield. In accordance with previously published data on healthy chondrocytes (Dozin et al. 2002), cell proliferation significantly decreased with increasing age at P1, but this significant correlation was not found between age and doubling rate at P2. A possible explanation can be found in the use of TGF $\beta 1$ and FGF2, growth factors able to increase the proliferation rate and to maintain the chondrocyte phenotype during the expansion procedures. This property is particularly useful when large amounts of cells are needed requiring a long-term expansion (Barbero et al. 2003). Thanks to the use of TGF $\beta 1$ and FGF-2, chondrocytes started soon to proliferate. The offset difference in cell proliferation rates that we found between $\mathrm{P} 1$ and $\mathrm{P} 2$ is in accordance with previous work (Barlic et al. 2008; Lin et al. 2006) and can be attributed to an initial lag phase where cells recover from isolation procedure. Interestingly, however, a relationship between the proliferation rate at $\mathrm{P} 1$ and at $\mathrm{P} 2$ was found in our data suggesting that cells with the lowest proliferation rate at $\mathrm{P} 1$ were also less proliferative at $\mathrm{P} 2$ and that the cells with high proliferation rate at P1 maintained this feature at $\mathrm{P} 2$. 
To correlate analyzed cellular parameters with the grade of cartilage degeneration, we evaluated random samples scored on the basis of ICRS scale. A lower cellular yield was observed in score 4 specimens. This result can be attributed to the higher degeneration level of this tissue whereby the inflammatory state of the tissue can affect cellularity. The proliferation rates for samples with different scores are in accordance with previously reported results (Yin et al. 2011): score has a slight influence only on the initial phase of in vitro expansion, whereas cell proliferation during the subsequent passages was independent from the degeneration of the excised cartilage.

In conclusion, our study allowed a systematic characterization of basic parameters as cellular yield and proliferation rates of chondrocytes originating from a wide group of OA patients. This characterization could be useful in view of a possible autologous cell therapy approach for osteoarthritis, as it is fundamental to determine the quality of the cell source, known to greatly influence the outcome of engineered tissue.

Acknowledgments Authors would like to acknowledge Dr. A. Degrate for his support in statistical analysis of data. This study was supported by Italian Ministry of Health.

\section{References}

Aigner T, Rose J, Martin J, Buckwalter J (2004) Aging theories of primary osteoarthritis: from epidemiology to molecular biology. Rejuvenation Res 7(2):134-145

Barbero A, Ploegert S, Heberer M, Martin I (2003) Plasticity of clonal populations of dedifferentiated adult human articular chondrocytes. Arthritis Rheum 48(5):1315-1325. doi: 10.1002/art.10950

Barbero A, Grogan S, Schafer D, Heberer M, Mainil-Varlet P, Martin I (2004) Age related changes in human articular chondrocyte yield, proliferation and post-expansion chondrogenic capacity. Osteoarthr Cartil 12(6):476-484

Barlic A, Drobnic M, Malicev E, Kregar-Velikonja N (2008) Quantitative analysis of gene expression in human articular chondrocytes assigned for autologous implantation. J Orthop Res 26(6):847-853

Bobacz K, Erlacher L, Smolen J, Soleiman A, Graninger WB (2004) Chondrocyte number and proteoglycan synthesis in the aging and osteoarthritic human articular cartilage. Ann Rheum Dis 63(12):1618-1622

Carossino AM, Recenti R, Carossino R, Piscitelli E, Gozzini A, Martineti V, Mavilia C, Franchi A, Danielli D, Aglietti P, Ciardullo A, Galli G, Tognarini I, Moggi Pignone A, Cagnoni M, Brandi ML (2007) Methodological models for in vitro amplification and maintenance of human articular chondrocytes from elderly patients. Biogerontology $8(5): 483-498$
Cavallo C, Desando G, Facchini A, Grigolo B (2010) Chondrocytes from patients with osteoarthritis express typical extracellular matrix molecules once grown onto a threedimensional hyaluronan-based scaffold. J Biomed Mater Res A 93(1):86-95

Cicuttini FM, Wluka AE, Wang Y, Stuckey SL, Davis SR (2003) Effect of estrogen replacement therapy on patella cartilage in healthy women. Clin Exp Rheumatol 21(1):79-82

Dehne T, Karlsson C, Ringe J, Sittinger M, Lindahl A (2009) Chondrogenic differentiation potential of osteoarthritic chondrocytes and their possible use in matrix-associated autologous chondrocyte transplantation. Arthritis Res Ther 11(5):R133

Dozin B, Malpeli M, Camardella L, Cancedda R, Pietrangelo A (2002) Response of young, aged and osteoarthritic human articular chondrocytes to inflammatory cytokines: molecular and cellular aspects. Matrix Biol 21(5):449-459

Goldring MB, Goldring SR (2007) Osteoarthritis. J Cell Physiol 213(3):626-634

Jakob M, Demarteau O, Schafer D, Stumm M, Heberer M, Martin I (2003) Enzymatic digestion of adult human articular cartilage yields a small fraction of the total available cells. Connect Tissue Res 44(3-4):173-180

Kleemann RU, Krocker D, Cedraro A, Tuischer J, Duda GN (2005) Altered cartilage mechanics and histology in knee osteoarthritis: relation to clinical assessment (ICRS grade). Osteoarthr Cartil 13(11):958-963

Lin Z, Willers C, Xu J, Zheng MH (2006) The chondrocyte: biology and clinical application. Tissue Eng 12(7):1971-1984

Loeser RF, Goldring SR, Scanzello CR, Goldring MB (2012) Osteoarthritis: a disease of the joint as an organ. Arthritis Rheum 64(6):1697-1707

Musumeci G, Loreto C, Carnazza ML, Martinez G (2011) Characterization of apoptosis in articular cartilage derived from the knee joints of patients with osteoarthritis. Knee Surg Sports Traumatol Arthrosc 19(2):307-313

Neri S, Mariani E, Cattini L, Facchini A (2011) Long-term in vitro expansion of osteoarthritic human articular chondrocytes do not alter genetic stability: a microsatellite instability analysis. J Cell Physiol 226(10):2579-2585

Roseti L, Bassi A, Grigolo B, Fornasari P (2011) Development of human chondrocyte-based medicinal products for autologous cell therapy. Biomaterials Science and Engineering. InTech

Tallheden T, Bengtsson C, Brantsing C, Sjogren-Jansson E, Carlsson L, Peterson L, Brittberg M, Lindahl A (2005) Proliferation and differentiation potential of chondrocytes from osteoarthritic patients. Arthritis Res Ther 7(3):R560R568

Tran-Khanh N, Hoemann CD, McKee MD, Henderson JE, Buschmann MD (2005) Aged bovine chondrocytes display a diminished capacity to produce a collagen-rich, mechanically functional cartilage extracellular matrix. J Orthop Res 23(6):1354-1362

van der Kraan PM, van den Berg WB (2008) Osteoarthritis in the context of ageing and evolution. Loss of chondrocyte differentiation block during ageing. Ageing Res Rev 7(2):106-113

Wang Y, Blasioli DJ, Kim HJ, Kim HS, Kaplan DL (2006) Cartilage tissue engineering with silk scaffolds and human articular chondrocytes. Biomaterials 27(25):4434-4442 
Wluka AE, Davis SR, Bailey M, Stuckey SL, Cicuttini FM (2001) Users of oestrogen replacement therapy have more knee cartilage than non-users. Ann Rheum Dis 60(4): 332-336

Yin J, Yang Z, Cao YP, Ge ZG (2011) Characterization of human primary chondrocytes of osteoarthritic cartilage at varying severity. Chin Med J (Engl) 124(24):4245-4253
Zhang W, Moskowitz RW, Nuki G, Abramson S, Altman RD, Arden N, Bierma-Zeinstra S, Brandt KD, Croft P, Doherty M, Dougados M, Hochberg M, Hunter DJ, Kwoh K, Lohmander LS, Tugwell P (2008) OARSI recommendations for the management of hip and knee osteoarthritis, part II: OARSI evidence-based, expert consensus guidelines. Osteoarthr Cartil 16(2):137-162 